\title{
Kernos
}

Revue internationale et pluridisciplinaire de religion grecque antique

33 | 2020

Varia

\section{Unlocking Sacred Landscapes}

Jean Vanden Broeck-Parant

\section{(2) OpenEdition \\ Journals}

\section{Édition électronique}

URL : https://journals.openedition.org/kernos/3514

DOI : 10.4000/kernos.3514

ISSN : 2034-7871

\section{Éditeur}

Centre international d'étude de la religion grecque antique

\section{Édition imprimée}

Date de publication : 31 décembre 2020

Pagination : $322-325$

ISBN : 978-2-87562-264-8

ISSN : 0776-3824

\section{Référence électronique}

Jean Vanden Broeck-Parant, « Unlocking Sacred Landscapes », Kernos [En ligne], 33 | 2020, mis en ligne le 31 décembre 2020, consulté le 06 décembre 2022. URL : http://journals.openedition.org/ kernos/3514; DOI : https://doi.org/10.4000/kernos.3514

Ce document a été généré automatiquement le 6 décembre 2022.

Tous droits réservés 


\title{
Unlocking Sacred Landscapes
}

\author{
Jean Vanden Broeck-Parant
}

\section{RÉFÉRENCE}

Giorgos Papantoniou, Christine E. moRris, Athanasios K. vionis (dir.), Unlocking Sacred Landscapes: Spatial Analysis of Ritual and Cult in the Mediterranean, Nicosie, Åström, 2019. 1 vol. 21,5 × 30,5 cm, 279 p. (Studies in Mediterranean Archaeology, 151).

1 Ce volume est la première publication du réseau de recherche Unlocking Sacred Landscape (UnSaLa), inauguré à l'occasion d'un séminaire international qui s'est tenu au Trinity College de Dublin en 2015. L'ouvrage comprend 15 contributions constituées en grande partie d'études présentées lors de ce séminaire, complétées par quelques études supplémentaires, et réparties en trois sections de tailles inégales. L'aire géographique couverte est vaste, s'étendant de Cadiz, à l'ouest, au Levant, à l'est, tandis que les périodes étudiées s'étalent de l'époque minoenne à la période ottomane. Un tel écart ne permet pas d'aborder toutes les régions ni toutes les périodes, tant s'en faut, et la période romaine et la Grèce archaïque et classique sont notablement absentes du sommaire, comme l'est d'ailleurs la Grèce continentale. Cette direction, qui n'est ni revendiquée, ni, semble-t-il, délibérée, permet de fait une exploration des paysages sacrés méditerranéens qui ne soit pas trop encombrée du poids des sources classiques. Certaines régions sont cependant mieux représentées que d'autres, au premier rang desquelles Chypre et la Crète, qui font respectivement l'objet de cinq et quatre contributions. Les autres lieux étudiés sont l'Italie centrale, Naxos, Constantinople et l'Idumée, tandis qu'un seul article adopte un point de vue transméditerranéen, par le prisme du monde phénico-punique.

2 Les éditeurs ambitionnent cependant d'étudier «l'espace rituel méditerranéen » de manière « holistique » (p. xiv), un programme à l'intention louable, mais aux contours incertains. L'espace méditerranéen n'est en effet pas défini et le choix de le traiter comme un ensemble n'est pas discuté; ce choix contraste d'ailleurs avec les études contenues dans le livre, qui se concentrent en majorité sur un site ou une région en 
particulier. Puisque le projet ne semble pas avoir non plus de limite temporelle (basse ou haute), il en résulte que l'approche holistique revendiquée par les auteurs demeure un concept assez flou. Néanmoins la volonté d'aborder le sacré en termes d'espaces (géographiques et/ou phénoménologiques) est claire et commune à tous les chapitres. L'angle principal du volume est méthodologique ; le lecteur aura pu s'en rendre compte par lui-même, mais ce n'est dit explicitement qu'au septième chapitre (p.121). L'objectif annoncé, en tout cas, est de "déverrouiller» ( unlock») les paysages sacrés et la religion en les replaçant dans leur contexte social et leur propre «longue durée ", en appliquant toutes les méthodes qui s'avèreront utiles. L'absence de contraintes temporelles ou méthodologiques assure certainement au projet de pouvoir englober une grande diversité de sujets, avec le risque, à plus long terme, de manquer de cap.

3 La première section (Sacred landscapes, socio-political units and socio-economic networks) reprend des études régionales ou, à tout le moins, inter-sites, dont la plupart font un usage important des méthodes avancées offertes par les SIG. G. Papantoniou enquête sur le rôle des sanctuaires dans la formation territoriale des entités politiques de Chypre aux époques archaïque et classique. Il s'appuie notamment sur les SIG, dont il teste ici l'utilité, et en particulier sur des analyses informatiques, désormais communes en archéologie, comme les calculs de visibilité (viewshed) ou d'itinéraire au moindre coût (least cost path). La conclusion de l'A. est pondérée : les SIG sont particulièrement utiles dans le cas d'études à échelle régionale, mais peuvent s'avérer problématiques lorsqu'il s'agit de les employer en diachronie ou sur des territoires plus vastes. En outre, les choix humains contredisent souvent les solutions les plus rationnelles telles que celles données par les SIG. Ces méthodes ne suffisent donc pas, à elles seules, à accéder au paysage mental des anciens.

4 L'étude de T. van Loon et T. de Haas sur le sanctuaire de Laghetto del Monsignore, dans le Latium, présente des préoccupations similaires à celles de G. Papantoniou : il s'agit notamment de dépasser les concepts de centre-périphérie et de liminalité traditionnellement appliqués aux sanctuaires (alors catégorisés comme urbains, suburbains ou extra-urbains), et d'analyser leur développement au cours du temps. La solution consiste à prendre comme point de départ de l'analyse le sanctuaire lui-même plutôt que le centre urbain dont il serait dépendant (ou non), et de le replacer dans son contexte local et régional. Il en résulte une image plus nuancée (quoique préliminaire) des différentes fonctions assurées par le sanctuaire de Laghetto del Monsignore à différentes périodes (de l'Âge du Fer à la période post-archaïque) et en particulier de son rapport avec le centre urbain proche de Satiricum.

N. Kyriakou insiste également sur l'importance de la longue durée dans son enquête sur le paysage religieux de Chypre durant l'Antiquité tardive (IV ${ }^{\mathrm{e}}-\mathrm{VII}{ }^{\mathrm{e}}$ siècles ap. J.-C.). L'A. analyse la distribution spatiale des lieux de culte chrétiens d'abord au sein des villes, puis à une échelle plus vaste, dans la " périphérie » de celles-ci. Par contraste avec les deux études précédentes, celle de $\mathrm{N}$. Kyriakou reprend donc à première vue la dialectique entre urbain et extra-urbain, une approche justifiée par l'organisation du territoire à l'époque romaine. Cependant l'analyse s'attarde plutôt sur les facteurs environnementaux qui expliquent l'implantation des basiliques. L'étude conclut que la distribution spatiale de celles-ci est l'expression de la mainmise des autorités ecclésiastiques sur les ressources agricoles et les réseaux commerciaux.

Il est encore question de distribution des églises dans l'article d'A.K. Vionis, plus particulièrement en milieu rural et entre les $\mathrm{x}^{\mathrm{e}}$ et $\mathrm{xvI}^{\mathrm{e}}$ siècles. Comme l'indique le titre, 
il s'agit d'une mise en parallèle (plutôt qu'une comparaison) de paysages religieux en mer Égée et à Chypre - en fait, à Naxos (surtout dans la partie centrale ouest de l'île) et dans la vallée de Soléa. Les raisons de rapprocher ces deux régions en particulier ne sont pas discutées de façon explicite. Une variété de méthodes est utilisée comme l'analyse des formes architecturales, l'iconographie et la cartographie, afin de mieux comprendre les divers rôles de ces églises comme marqueurs dans l'espace. On retient notamment que l'analyse tend à confirmer l'organisation de l'habitat décrite par les sources textuelles.

7 S. Turner et J. Crow discutent de différentes méthodes digitales pour aborder le paysage religieux de Naxos entre 600 et 1000 ap. J.-C., sur base de quelques cas d'étude. L'enregistrement 3D au moyen d'un scanneur laser produit un enregistrement permanent d'une structure qui peut servir à une étude architecturale plus approfondie. Les A. présentent également une méthode basée sur les SIG et baptisée Historic Landscape Characterisation qui consiste à interpréter le paysage dans sa totalité, sous une forme continue (c'est-à-dire sans espaces « vides » sur la carte) plutôt que ponctuelle, et qui permet de mieux comprendre, dans le cas présent, l'emplacement des églises.

Le chapitre offert par L. Nixon est plus circonscrit, puisqu'il se focalise sur la ville crétoise de Chania et sa transformation suite à la conquête ottomane de 1645. Il apparaît que les changements apportés au paysage religieux furent relativement minimes, mais ciblés, dans un effort stratégique visant à affirmer rapidement la nouvelle identité ottomane de la ville. L'A. propose ensuite deux pistes de recherche : «l'ottomanisation " (au travers de l'étude des toponymes) et la « dé-ottomanisation » de la Crète.

La deuxième section du livre (Experiencing sacred landscapes) rassemble trois contributions qui ont en commun d'aborder la question de la perception subjective de l'espace rituel.

10 L. Goodison propose ainsi d'analyser les tombes collectives du type Mesara, construites en Crète entre 3500 et 1650 av. J.-C. environ, en termes "d'espace vécu », par trois biais : la «topographie » (qui inclut le ciel, le paysage et le corps humain), le temps, et la conjonction de ceux-ci, la «transaction ». Les données archéologiques suggèrent une architecture à fonction exégétique répondant à des croyances liées à la vie et à la mort, un alignement avec le lever du soleil à des moments spécifiques de l'année, ainsi qu'un désir d'interaction avec les défunts manifestée par la manipulation des ossements.

11 Ce souhait de donner une plus grande place à l'émotion et à l'expérience sous-tend également l'article de M.Lopez-Bertran sur l'importance des sens dans les rituels funéraires dans le monde phénico-punique entre 900 et 200 av. J.-C. Le chapitre est organisé selon les cinq sens, mais l'A. souligne également l'importance, dans ce type de contextes, de la synesthésie et du « sens du lieu » qui devait se créer à l'occasion de ces rituels, autant d'expériences sensorielles qui échappent à notre classification traditionnelle des sens.

12 Enfin, V. Manolopoulou explore les dimensions spatiale et temporelle des processions religieuses dans la Constantinople byzantine. La densité de fréquentation de certaines églises renvoie à des événements historiques de la ville, tandis que l'usage de certains sites a pu être dicté par les saisons. D'un point de vue méthodologique, l'A. souhaite montrer l'utilité des outils digitaux, en particulier des SIG, pour approcher les rituels et le paysage d'un point de vue spatiotemporel. 
13 La troisième et dernière section reprend les contributions liées à la question de l'agentivité et des artefacts dans l'espace sacré, c'est-à-dire des actions rituelles et des objets qui leur sont associés.

Ce sont précisément ces thèmes que $\mathrm{M}$. Webb aborde dans son article sur les tombes chypriotes de l'Âge du Bronze, en partant du principe d'une corrélation entre le degré de contestation de ces espaces et l'importance des rituels et paraphernalia qui leur sont associés. À partir de deux cas d'étude sur la côte nord de l'île, l'A. enquête sur les relations de pouvoir intra- et inter-sites, dans le contexte du développement d'entités politiques.

15 A. Peatfield et Chr. Morris, quant à eux, proposent une double analyse du sanctuaire de sommet minoen d'Astipadhes Korakias, en tant que lieu (donc essentiellement en termes d'accessibilité et de visibilité) et en tant qu'espace. La distribution intra-site des trouvailles archéologiques, en particulier les figurines en terre cuite, suggère des actions rituelles différenciées selon les subdivisions de cet espace. On regrette un peu que cette belle enquête se termine de façon si abrupte sur une courte réflexion, pourtant intéressante, sur le rapport entre paysage et cosmologie, et qui fait remarquablement écho à l'article signé par L. Goodison.

16 Il est encore question de figurines, à Chypre cette fois, dans la contribution de C. Alexandrou, qui se concentre sur les figurines féminines du type "Base-Ring", produites entre les $\mathrm{XV}^{\mathrm{e}}$ et $\mathrm{XII}^{\mathrm{e}}$ siècles av. J.-C. L'étude se focalise sur les figurines trouvées en contexte domestique, mieux documentées que celles provenant de contextes funéraires ou rituels. Passant d'une analyse spatiale intra-site à inter-sites, l'A. examine les lieux de découverte, les objets associés à ces figurines et l'aspect fragmentaire de certaines d'entre elles. Les conclusions sont encore fragiles, mais, malgré le caractère incomplet des données, il apparaît qu'une faible proportion de figurines a pu faire l'objet d'un dépôt ritualisé.

17 On revient dans l'Italie de l'Âge du Fer avec l'étude de J. Tabolli sur le paysage funéraire de La Petrina, dans l'Ager Faliscus. En combinant différentes méthodes et données, il propose une analyse spatiale de la nécropole qui suit la route, à peine mentionnée dans le rapport de fouilles de la fin du $\mathrm{XIX}^{\mathrm{e}}$ siècle, qui articulait les trois terrasses sur lesquelles s'étendait le cimetière. Le paysage funéraire ainsi reconstitué dévoile un tissu complexe d'actions rituelles aux niveaux individuel, familial et communautaire, et témoigne de l'importance de cet espace pour la représentation politique de la communauté de Narce, à l'intérieur comme à l'extérieur.

G. Papasavvas réfléchit quant à lui au développement des États en Crète de l'Âge du Fer au travers du cas du sanctuaire « extra-urbain » d'Hermès et Aphrodite à Symè, associé à des rites de passage. L'abondance et la richesse du matériel votif, ainsi que le caractère monumental de la terrasse, qui a dû requérir la coordination de plusieurs communautés voisines, attestent l'importance de ce sanctuaire. Papasavvas remet en cause l'idée selon laquelle l'une de ces communautés en avait pris le contrôle exclusif au début du $\mathrm{VII}^{\mathrm{e}}$ siècle. Il conclut que, par contraste avec la Grèce continentale, ce n'est pas l'expansion des grands sanctuaires non urbains qui a contribué à l'émergence des poleis mais, au contraire, leur déclin, associé à la relocalisation des lieux de culte à l'intérieur des zones d'habitat.

19 Le volume se conclut avec un article d'A. Erlich qui compare les assemblages de figurines en terre cuite de trois sites de l'Idumée, entre la conquête perse et le début de 
l'époque hellénistique. La présence nettement plus importante des types iduméens au site de Maresha s'expliquerait non pas tant par la position hiérarchique du site que par une volonté d'y manifester une identité collective.

20 Les éditeurs souhaitaient fournir un ouvrage utile pour l'étude des paysages sacrés méditerranéens. La variété des approches qui y sont présentées donne en tout cas matière à réflexion et certaines méthodes gagneront à être testées (moyennant, sans doute, quelques ajustements) dans d'autres contextes.

\section{AUTEURS}

\section{JEAN VANDEN BROECK-PARANT}

Université d'Utrecht 